\title{
Analysis of Asymptomatic and Presymptomatic Transmission in SARS-CoV-2 Outbreak, Germany, 2020
}

\author{
Jennifer K. Bender, ${ }^{1}$ Michael Brandl, ${ }^{1}$ Michael Höhle, Udo Buchholz, Nadine Zeitlmann
}

We determined secondary attack rates (SAR) among close contacts of 59 asymptomatic and symptomatic coronavirus disease case-patients by presymptomatic and symptomatic exposure. We observed no transmission from asymptomatic case-patients and highest SAR through presymptomatic exposure. Rapid quarantine of close contacts with or without symptoms is needed to prevent presymptomatic transmission.

$\mathrm{D}$ uring the ongoing coronavirus disease (COVID-19) pandemic, worldwide, >85 million severe acute respiratory syndrome coronavirus 2 (SARSCoV-2) infections had been reported as of January 7, 2021 (https://covid19.who.int). Although it was clear from the beginning of the pandemic that symptomatic transmission of SARS-CoV-2 occurs, presymptomatic transmission has also been described (1-6). Furthermore, transmission from asymptomatic cases was deemed possible on the basis of findings that viral load of asymptomatic cases was similar to that of symptomatic cases (7). Understanding how transmission occurs from asymptomatic cases and from symptomatic cases in their presymptomatic and symptomatic phase, as well as the frequency of transmission, is essential for public health management. We assessed asymptomatic, presymptomatic, and symptomatic transmission during an outbreak investigation of 59 COVID-19 cases by determining secondary attack rates (SAR) according to the

Author affiliations: Robert Koch Institute, Wernigerode, Germany (J.K. Bender); European Centre for Disease Prevention and Control, Stockholm, Sweden (J.K. Bender, M. Brandl); Robert Koch Institute, Berlin, Germany (M. Brandl, M. Höhle, U. Buchholz, N. Zeitlmann); Stockholm University, Stockholm (M. Höhle); Federal Institute for Quality Assurance and Transparency in Healthcare, Berlin (M. Höhle)

DOI: https://doi.org/10.3201/eid2704.204576 respective exposure periods. In addition, we estimated key parameters such as serial interval and incubation period.

\section{The Study}

On February 29, 2020, a COVID-19 case was notified to the local public health authority (LPHA) of a rural district in southern Germany without previously observed community transmission. During the infectious period, the case-patient had attended several carnival events in the district. The LPHA immediately initiated contact tracing, identifying all close contacts; they were quarantined and tested irrespective of symptoms. By the end of March 2020, a cluster of 59 cases had been identified through successive contact tracing activities.

We interviewed the case-patients of the cluster by phone regarding symptoms developed during SARS-CoV-2 infection; potential source cases or events; and household contacts (HCs) and close nonhousehold or other contacts (OCs) in their infectious period (Appendix, https://wwwnc.cdc.gov/ EID/article/27/4/20-4576-App1.pdf). We obtained an empirical distribution of the serial interval from the average over all possible transmission trees of the cluster. We obtained generation time and incubation period by averaging over the estimates as described by Reich et al. (8) (Appendix).

To estimate SAR and relative risks (RRs) we conducted a retrospective cohort study, including all HCs and OCs as recalled by the case-patients that met inclusion criteria (Appendix). We calculated pooled SAR of HCs and OCs for 2 outcomes, laboratory confirmation $\left(\mathrm{SAR}_{\mathrm{lab}}\right)$ and development of respiratory symptoms $\left(\mathrm{SAR}_{\mathrm{res}}\right)$ in the following groups: HCs and OCs of asymptomatic case-patients who never experienced symptoms; HCs and OCs of

\footnotetext{
${ }^{1}$ These first authors contributed equally to this article.
} 
Table 1. Demographics of coronavirus disease case-patients and their contacts in a district in southern Germany*

\begin{tabular}{|c|c|c|c|c|c|}
\hline \multirow[b]{2}{*}{ Case type } & \multirow[b]{2}{*}{$\begin{array}{c}\text { No. }(\%) \\
\text { asymptomatic }\end{array}$} & \multicolumn{3}{|c|}{ No. (\%) symptomatic } & \multirow[b]{2}{*}{ Total } \\
\hline & & $\begin{array}{l}\text { Phase not specified } \\
\text { or both } \dagger\end{array}$ & $\begin{array}{l}\text { Presymptomatic } \\
\text { phase only }\end{array}$ & $\begin{array}{l}\text { Symptomatic } \\
\text { phase only }\end{array}$ & \\
\hline \multicolumn{6}{|l|}{ Case-patients } \\
\hline Total & $7(13.2)$ & $46(86.8)$ & NA & NA & $53(100)$ \\
\hline Female & 3 (11.5) & 23 (88.5) & NA & NA & $26(100)$ \\
\hline Male & $4(14.8)$ & $23(85.2)$ & NA & NA & 27 (100) \\
\hline Median age & 36 (IQR 6-68) & 40 (IQR 29-50) & NA & NA & 39.5 (IQR 29-50)‡ \\
\hline \multicolumn{6}{|c|}{ Contact persons by type of exposure } \\
\hline $\mathrm{HC}$ & $7(16.7)$ & $35(83.3)$ & NA & NA & $42(100)$ \\
\hline $\mathrm{OC}$ & $52(24.5)$ & $48(22.6)$ & $81(38.2)$ & $31(14.6)$ & $212(100)$ \\
\hline
\end{tabular}

*HC, household contact; IQR, interquartile range; NA, not applicable; OC, nonhousehold or other contact.

†The phase in which the contact occurred was not specified, or contact occurred in both phases.

$\ddagger$ Three of 53 cases were children $<15$ y of age.

symptomatic case-patients in which the phase with contact could not be specified by the case-patient or with contact in both phases; OCs of symptomatic case-patients with contact only in the presymptomatic phase; and OCs of symptomatic case-patients with contact only in the symptomatic phase.

We were able to contact 53/59 (90\%) case-patients. Three case-patients were children $<15$ years of age (Table 1). Forty-six (87\%) were symptomatic, and $7(13 \%)$ were asymptomatic (Appendix Figure 1). The cluster resulted in 144 possible transmission trees, which span over 5 generations (Figure). No secondary transmission resulted from asymptomatic cases. We determined a median serial interval of 3.0 (IQR 1.0-6.0) days and a median incubation period of 4.3 (IQR 2.5-6.5) days (Appendix Table 1).

In total, $42 \mathrm{HCs}$ and 212 OCs were included in the cohort study (Table 1$)$. The overall SAR lab $_{\text {was }} 13 \%$ (4/32) for HCs and 14\% (20/148) for OCs. The overall SAR ${ }_{\text {res }}$ was $29 \%(12 / 42)$ for HCs and $17 \%(29 / 170)$

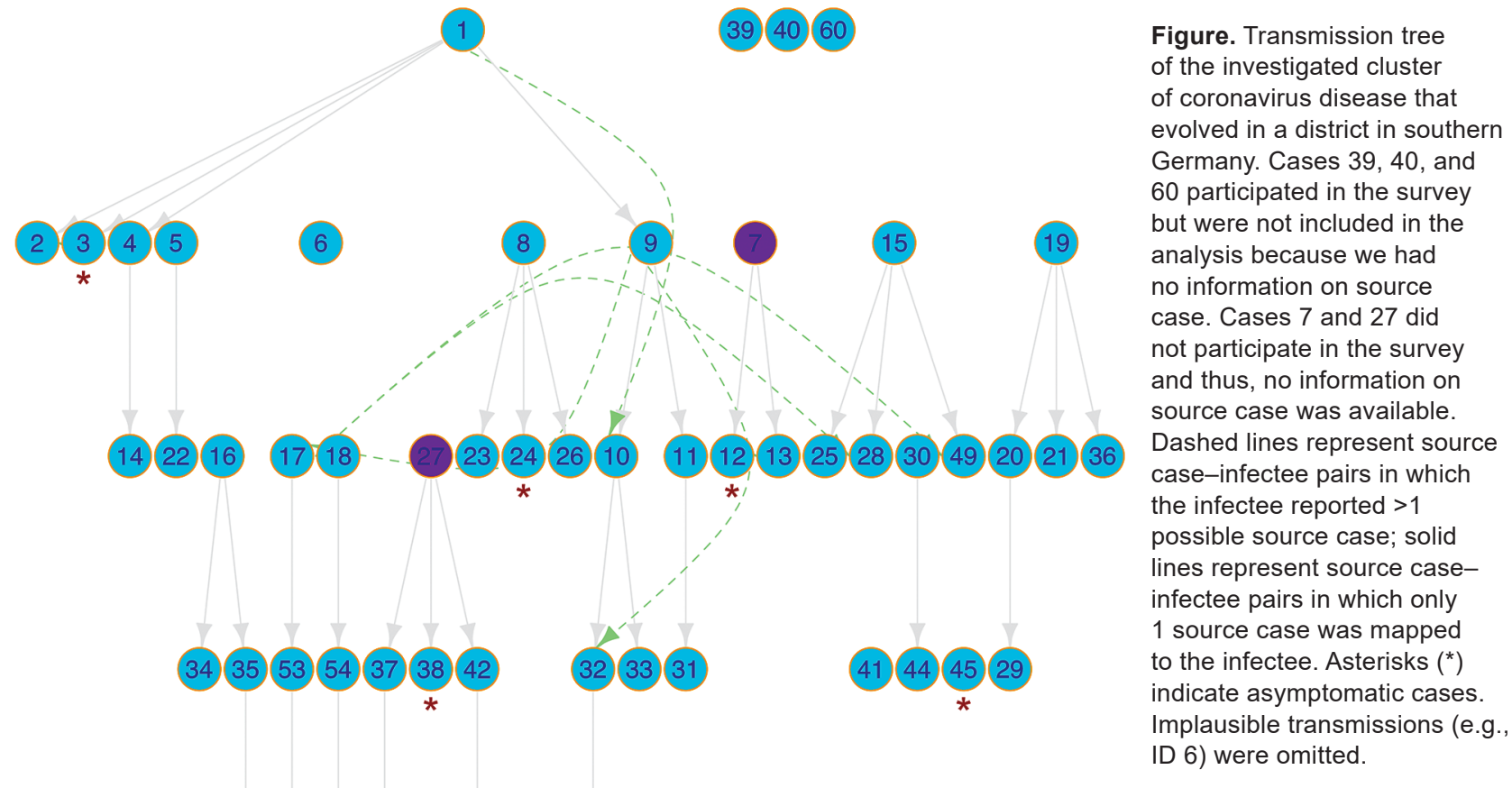

O With survey info

- Without survey info 
Presymptomatic Transmission of SARS-CoV-2

Table 2. Secondary attack rates among contacts of coronavirus disease case-patients in a district in southern Germany*

\begin{tabular}{|c|c|c|c|c|}
\hline Clinical symptoms of source case & $\begin{array}{l}\text { No. contacts tested positive or } \\
\text { experienced respiratory symptoms }\end{array}$ & $\begin{array}{l}\text { Total no. } \\
\text { contacts }\end{array}$ & SAR, \% & $\mathrm{RR}(95 \% \mathrm{Cl})$ \\
\hline \multicolumn{5}{|l|}{ Household contacts SAR lab } \\
\hline Asymptomatic & 0 & 4 & 0 & \multirow{3}{*}{$\begin{array}{c}\text { Reference } \\
0.8(0.09-\infty)\end{array}$} \\
\hline Symptomatic, phase not specified or both $\dagger$ & 4 & 28 & 14.3 & \\
\hline Total & 4 & 32 & 12.5 & \\
\hline \multicolumn{5}{|l|}{ Household contacts SAR res } \\
\hline Asymptomatic & 0 & 7 & 0 & \multirow{3}{*}{$\begin{array}{c}\text { Reference } \\
3.4(0.56-\infty)\end{array}$} \\
\hline Symptomatic, phase not specified or both & 12 & 35 & 34.3 & \\
\hline Total & 12 & 42 & 28.6 & \\
\hline \multicolumn{5}{|l|}{ Other contacts SAR $\mathrm{R}_{\text {lab }}$} \\
\hline Asymptomatic cases & 0 & 22 & 0 & \multirow{5}{*}{$\begin{array}{c}\text { Reference } \\
3.4(0.36-\infty) \\
6.5(1.1-\infty) \\
1.8(0.14-\infty)\end{array}$} \\
\hline Symptomatic, phase not specified or both & 3 & 25 & 12.0 & \\
\hline Symptomatic, presymptomatic phase only & 15 & 72 & 20.8 & \\
\hline Symptomatic, symptomatic phase only & 2 & 29 & 6.9 & \\
\hline Total & 20 & 148 & 13.5 & \\
\hline \multicolumn{5}{|l|}{ Other contacts SAR res } \\
\hline Asymptomatic cases & 2 & 52 & 3.8 & Reference \\
\hline Symptomatic, phase not specified or both & 4 & 22 & 18.2 & $4.7(0.68-52)$ \\
\hline Symptomatic, presymptomatic phase only & 22 & 67 & 32.8 & $8.5(2.1-75)$ \\
\hline Symptomatic, symptomatic phase only & 1 & 29 & 3.5 & $0.90(0.02-17)$ \\
\hline Total & 29 & 170 & 17.1 & \\
\hline
\end{tabular}

for OCs (Table 2). We did not identify any HC who tested positive or experienced respiratory symptoms after contact with asymptomatic case-patients. Neither $\mathrm{SAR}_{\mathrm{lab}}$ nor SAR res of HCs of symptomatic casepatients were significantly higher compared with HCs of asymptomatic cases $\left(\mathrm{SAR}_{\mathrm{lab}} \mathrm{p}=1.0\right.$; $\mathrm{SAR}_{\mathrm{res}} \mathrm{p}$ $=0.23$ ). We observed no laboratory-confirmed SARSCoV-2 transmission from asymptomatic case-patients to any of the 22 OCs (Table 2; Appendix Figure 2). SAR $_{\text {lab }}$ was highest for OCs with contact during the case-patients' presymptomatic phases $(21 \% ; 15 / 72)$ yielding a RR of 6.5 (95\% CI 1.1- $\infty)$ when compared with contacts of asymptomatic case-patients. Adjusting for case-patients' age, sex, and number of contact persons showed no substantial changes in the magnitude of estimates (data not shown). Presymptomatic transmission accounted for $\geq 75 \%$ of all transmissions to OCs in the cohort (Appendix).

\section{Conclusions}

In this cluster of COVID-19 cases, little to no transmission occurred from asymptomatic case-patients. Presymptomatic transmission was more frequent than symptomatic transmission. The serial interval was short; very short intervals occurred.

The fact that we did not detect any laboratory-confirmed SARS-CoV-2 transmission from asymptomatic case-patients is in line with multiple studies (9-11). However, Oran et al. have speculated that asymptomatic cases contribute to the rapid progression of the pandemic (12). Some studies may be prone to misclassify presymptomatic cases as asymptomatic, leading to heterogeneous reporting of SAR of asymptomatic cases, because of different case definitions or differential duration of follow-up. In our study we used a very sensitive case definition for symptomatic cases that did not require specific symptoms (e.g. fever) to be present. Also, timing of our study would have enabled detection of late onset of symptoms, which gives us confidence in our classification of exposure groups.

The $75 \%$ of SARS-CoV-2 transmissions in our cohort from case-patients in their presymptomatic phase exceeds reported transmission rates from other investigations $(1,13,14)$. Possible reasons are the prior evidence that infectiousness peaks around the date of symptom onset, declining thereafter (15), and that case-patients probably reduced social contacts themselves once they experienced symptoms or when ordered to self-isolate. A large proportion of cases with presymptomatic transmission in our cluster is further supported by the median serial interval of 3 days.

Of note are the consequences for public health management: first, the need for early detection of COVID-19 cases and for initiation of contact tracing as soon as possible to quarantine close contacts, particularly because short serial intervals may lead to further transmission chains. Second, suspect case-patients or persons with any respiratory illness should immediately self-isolate and inform their contacts met in the presymptomatic and symptomatic phases.

A limitation of our study is that evidence was obtained from a single outbreak and might not be 
applicable to other settings. We used only information as recalled by the case-patients, which is imperfect and may introduce errors or bias. Because we used development of respiratory symptoms as a proxy for possible SARS-CoV-2 infections among contacts, and because incidence of respiratory illnesses was still high in this winter

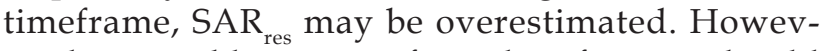
er, this possible source of misclassification should be nondifferential between groups. We excluded many HCs because of uncertainties about the potential simultaneous introduction of SARS-CoV-2 in the household, which may have led to an underestimation of SAR among HCs. In the transmission tree, we had to omit various source case-infectee pairs because case-patients' recalled symptom onset differed substantially from surveillance data and was not plausible (Appendix). Finally, although community transmission of SARS-CoV-2 was deemed unlikely in the affected district at the time, we cannot rule out that some cases acquired infections from other sources.

In conclusion, our study suggests that asymptomatic cases are unlikely to contribute substantially to the spread of SARS-CoV-2. COVID-19 cases should be detected and managed early to quarantine close contacts immediately and prevent presymptomatic transmissions.

\section{Acknowledgments}

As J.K.B. is a fellow of the European Public Health Microbiology Training at the European Centre for Disease Prevention and Control (ECDC) Fellowship Programme (and supported financially by this program) and M.B. is a fellow of the European Programme for Intervention Epidemiology Training at the ECDC and the Postgraduate Training for Applied Epidemiology at the Robert Koch Institute, we first acknowledge these programs. We also thank the local public health authority of the district for its continuous efforts fighting the COVID-19 pandemic as well as for providing us with information about the cluster and corresponding data for subsequent analysis. We thank the team of researchers assisting with the case interviews: Johannes Zeiher, Nora-Katharina Küpke, Sandra Niendorf, Sangeeta Banerji, and Susann Dupke. Furthermore, we especially thank all COVID-19 case-patients of this cluster for their participation in the study.

\section{About the Author}

Dr. Bender is a fellow of the European Public Health Microbiology Training Programme of the European Centre for Disease Prevention and Control. She is a microbiologist affiliated with the Nosocomial Pathogens and Antibiotic Resistances Unit, Department of Infectious Diseases of the Robert Koch Institute. Her research interest focuses on the emergence of multidrug-resistant nosocomial pathogens.

\section{References}

1. Wei WE, Li Z, Chiew CJ, Yong SE, Toh MP, Lee VJ. Presymptomatic transmission of SARS-CoV-2 - Singapore, January 23-March 16, 2020. MMWR Morb Mortal Wkly Rep. 2020;69:411-5. https:// doi.org/10.15585/mmwr.mm6914e1

2. Böhmer MM, Buchholz U, Corman VM, Hoch M, Katz K, Marosevic DV, et al. Investigation of a COVID-19 outbreak in Germany resulting from a single travel-associated primary case: a case series. Lancet Infect Dis. 2020;20:920-8. https://doi.org/10.1016/S1473-3099(20)30314-5

3. Tong ZD, Tang A, Li KF, Li P, Wang HL, Yi JP, et al. Potential presymptomatic transmission of SARS-CoV-2, Zhejiang Province, China, 2020. Emerg Infect Dis. 2020;26:1052-4. https://doi.org/10.3201/eid2605.200198

4. Huang L, Zhang $X$, Zhang X, Wei Z, Zhang L, Xu J, et al. Rapid asymptomatic transmission of COVID-19 during the incubation period demonstrating strong infectivity in a cluster of youngsters aged 16-23 years outside Wuhan and characteristics of young patients with COVID-19: A prospective contact-tracing study. J Infect. 2020;80:e1-13. https://doi.org/10.1016/j.jinf.2020.03.006

5. Yu P, Zhu J, Zhang Z, Han Y. A familial cluster of infection associated with the 2019 novel coronavirus indicating possible person-to-person transmission during the incubation period. J Infect Dis. 2020;221:1757-61. https:/ / doi.org/10.1093/infdis/jiaa077

6. Arons MM, Hatfield KM, Reddy SC, Kimball A, James A, Jacobs JR, et al.; Public Health-Seattle and King County and CDC COVID-19 Investigation Team. Presymptomatic SARS-CoV-2 infections and transmission in a skilled nursing facility. N Engl J Med. 2020;382:2081-90. https://doi.org/10.1056/NEJMoa2008457

7. Zou L, Ruan F, Huang M, Liang L, Huang H, Hong Z, et al. SARS-CoV-2 viral load in upper respiratory specimens of infected patients. N Engl J Med. 2020;382:1177-9. https://doi.org/10.1056/NEJMc2001737

8. Reich NG, Lessler J, Cummings DA, Brookmeyer R. Estimating incubation period distributions with coarse data. Stat Med. 2009;28:2769-84. https:// doi.org/10.1002/ sim.3659

9. Park SY, Kim Y-M, Yi S, Lee S, Na B-J, Kim CB, et al. Coronavirus disease outbreak in call center, South Korea. Emerg Infect Dis. 2020;26:1666-70. https:/ / doi.org/10.3201/ eid2608.201274

10. Cheng HY, Jian SW, Liu DP, Ng TC, Huang WT, Lin HH; Taiwan COVID-19 Outbreak Investigation Team. Contact tracing assessment of COVID-19 transmission dynamics in Taiwan and risk at different exposure periods before and after symptom onset. JAMA Intern Med. 2020;180:1156-63. https:/ / doi.org/10.1001/jamainternmed.2020.2020

11. Luo L, Liu D, Liao X, Wu X, Jing Q, Zheng J, et al. Contact settings and risk for transmission in 3,410 close contacts of patients with COVID-19 in Guangzhou, China. Ann Intern Med. 2020;173:879-87. https:/ / doi.org/10.7326/ M20-2671

12. Oran DP, Topol EJ. Prevalence of asymptomatic SARS-CoV-2 infection: a narrative review. Ann Intern Med. 2020;173: 362-7. https:/ / doi.org/10.7326/M20-3012 
13. Ferretti L, Wymant C, Kendall M, Zhao L, Nurtay A, AbelerDörner L, et al. Quantifying SARS-CoV-2 transmission suggests epidemic control with digital contact tracing. Science. 2020;368:eabb6936. https:// doi.org/10.1126/science.abb6936

14. Ganyani T, Kremer C, Chen D, Torneri A, Faes C, Wallinga J, et al. Estimating the generation interval for coronavirus disease (COVID-19) based on symptom onset data, March 2020. Euro Surveill. 2020;25. https:/ / doi.org/10.2807/ 1560-7917.ES.2020.25.17.2000257
15. He X, Lau EHY, Wu P, Deng X, Wang J, Hao X, et al. Temporal dynamics in viral shedding and transmissibility of COVID-19. Nat Med. 2020;26:672-5. https:/ / doi.org/ $10.1038 / \mathrm{s} 41591-020-0869-5$

Address for correspondence: Jennifer K. Bender, Department of Infectious Diseases, Robert Koch Institute, Burgstrasse 37, 38855 Wernigerode, Germany; email: benderj@rki.de

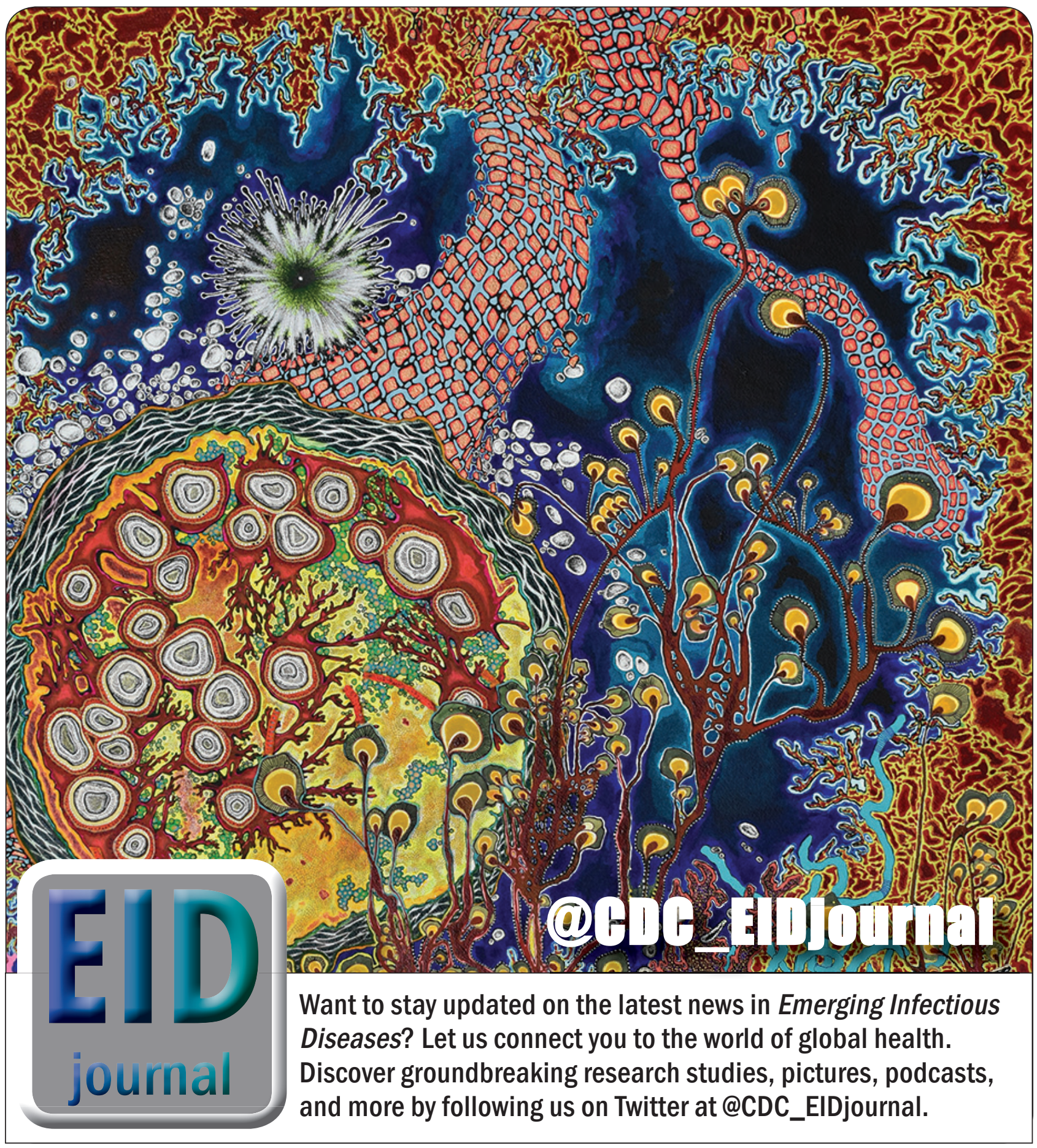

